\title{
Synthesis of protocatechuic acid-zinc/aluminium-layered double hydroxide nanocomposite as an anticancer nanodelivery system
}

\begin{abstract}
Protocatechuic acid, an active anticancer agent, has been intercalated into $\mathrm{Zn} / \mathrm{Al}$-layered double hydroxide at $\mathrm{Zn} / \mathrm{Al}=2$ ) using two different preparation methods, co-precipitation and ion-exchange, which are labelled as PZAE and PZAC, respectively. The release of protocatechuate from the nanocomposites occurred in a controlled manner and was fitted satisfactorily to pseudo-second order kinetics. The basal spacing of the resulting nanocomposites PZAE and PZAC was 10.2 and $11.0 \AA$, respectively, indicating successful intercalation of protocatechuate anions into the interlayer galleries of $\mathrm{Zn} / \mathrm{Al}-\mathrm{NO} 3-\mathrm{LDH}$ in a monolayer arrangement with angles of 24 and $33^{\circ}$ from the z-axis in PZAE and PZAC, respectively. The formation of nanocomposites was further confirmed by a Fourier transform infrared study. Thermogravimetric and differential thermogravimetric analyses indicated that the thermal stability of the intercalated protocatechuic acid was significantly enhanced compared to its free protocatechuic acid, and the drug content in the nanocomposites was estimated to be approximately $32.6 \%$ in PZAE and 29.2\% in PZAC. Both PZAE and PZAC nanocomposites inhibit the growth of human cervical, liver and colorectal cancer cell lines and exhibit no toxic effects towards normal fibroblast $3 \mathrm{~T} 3$ cell after $72 \mathrm{~h}$ of treatment.
\end{abstract}

Keyword: Protocatechuic acid; Zn/Al-LDH; 3T3; HeLa; HT29; HepG2 cell lines 